\title{
Study on the Preliminary Construction of the Cloud of Mental Health Education in Chinese Colleges and Universities
}

\author{
Liu Shengyue $e^{1}$ \\ School of Political Science and Public Administration, \\ University of Electronic Science and Technology of China, \\ Chengdu, P.R.China, 611731 \\ chrisour@163.com \\ Li Yuan* \\ Mental Health Education Center, \\ University of Electronic Science and Technology of China, \\ Chengdu, P.R.China, 611731 \\ liyuan@uestc.edu.cn
}

\author{
Zeng Weixi ${ }^{2}$ \\ School of Political Science and Public Administration, \\ University of Electronic Science and Technology of China, \\ Chengdu, P.R.China, 611731 \\ zcj213@126.com
}

\begin{abstract}
In recent years, the combination of network and mental health education in colleges has realized its transition from the tool and problem consciousness period to the system construction period. However, some shortcomings still exist in the system construction period: the resources integration of mental health education is insufficient; such resources are not shared among colleges; the network mental health education has sole function. Recently, the wide use of Cloud technology indicates such combination of network and mental health education will be expanded. Cloud technology make it possible to overcome these shortcomings. To make the Cloud technology and the mental health education combine well, the author proposes a new concept, The Cloud of Mental Health Education in colleges and universities, and discuss its practical significance and value. Besides, this study makes a preliminary construction for the Cloud from the aspects of platforms and services which may contribute to practical operation.
\end{abstract}

Keywords-colleges and universities; mental health education; Cloud computing; construction

\section{INTRODUCTION}

The Chinese scholars early define the relationship between network and mental health education from the perspective of tool consciousness and problem consciousness. In this phase, network has been used as a technology and a platform for information exchange. Therefore, network has been used in mental health education such as online psychological training, counseling, testing, and treatment. In the second phase, the combination scholars concerned becomes different. Network has not only been treated as an extension of the traditional mental health education, but the networking of the mental health education, that is, the formation of the network system of mental health education. Although the domestic colleges and universities do pay attention to the construction of the network system of mental health education, say, making psychological webpages and developing resources, there are also some problems. Firstly, the focus of function design has been put on preventing and resolving the mental problems college students may encounter during the process of growing up. For instance, network platforms have been used to make an appointment of psychological counseling. It neglects the communication and interaction with users and doesn't achieve the function of collecting, classifying and integrating the data of college students' mental health via network platforms. Secondly, that content construction mainly focuses on releasing the information and news of college students' mental health activities doesn't meet the needs of mental health education for them [1]. It fails to provide the resources for science popularization and education and build a self-help learning system to supply personalized mental health services to students. Thirdly, the mechanism of co-sharing and coconstruction has not been completely established among colleges and universities. That the platforms of each college haven't been linked as a web leads to the information islands. For another hand, in view of lack of standardized design, that the data exchange are limited by copyright protection, the editions of software and bandwidth results in some difficulties such as low access speed, long download time and low utilization ratio.

Zeng Wenxiong (2012) insists that Cloud computing technology can cope with the problems above. Cloud computing technology, the integrated derivation of distributed processing, parallel processing, network storage and hot backup redundancy and other network technologies, is an available, convenient, on-demand network access mode with extraordinary function of computing, storaging, high security and high compatibility [2]. The shared pool of configurable computing resources (e.g., The network server, storage equipment, selection of network equipment and apps) is easily be accessed by could computing technology that can make 
resources rapidly provided to satisfy the need of the users. It can realize the integration of the mental education resources and ensure the safety and speed of the access to the personalized mental health education services. Therefore, it is significant to combine mental health education with Cloud computing technology. However, there are few related researches in this field at present, this paper come up with a new concept names the Cloud of Mental Health Education in Colleges and Universities. Its practical value and elements will be discussed in the following sections.

\section{DEFINITION}

the Cloud of Mental Health Education in Colleges and Universities which has been proposed based on the Cloud computing technology's fantastic computing and storaging capacity and its heterogeneous-access and sharing, is a tremendous data pool which can integrate all the mental health education resources among colleges and universities. It can not only realize the resources sharing among colleges but provided users with reliable custom services of mental health education resources in Chinese colleges.

\section{THE SIGNIFICANCE OF BUILDING THE CLOUD OF MENTAL HEALTH EDUCATION IN COLLEGES AND UNIVERSITIES}

\section{A. It is the requirement of the era}

For college students, the network is a double-edged sword which has powerful attractiveness and inevitable realistic perplexities. Along with the era of cloud and digitalization, the Internet has played a indispensable role in scientific research, learning skills, knowledge acquisition and interpersonal communication. Mental health education will be disconnected with the era if it doesn't catch up with the step of Cloud Era and only sticks to the traditional developing model. Therefore, it is urgent to explore a system of network mental health education which is suitable for the initial needs of the college students.

\section{$B$. It is the requirement of resolving Internet-related mental problems}

At present, the academic community argues that the content of the mental health education has two aspects: disseminating the basic knowledge of mental health and solving the mental problems [3]. the Cloud of Mental Health Education in Colleges and Universities is serve for network mental health education and its partial function is to analysis the time segments and preference of the internet usage, the ratio of network resources and the group network behavior of college students by using Cloud computing technology. It can localize and screen the individuals who has mental problems such as Digital Personality Disorder, Paranoia in communication with machine and Network Demoralization Symptoms, etc.

\section{It is the requirement of educational innovation}

In the era of Cloud, The subject of education is becoming more and more non subjective. That is to say, it is no longer the authority to instill ideas, but the subject which can produce, disseminate, storage and monitor the network information.
Therefore, it has three identity of the thought leader, the information disseminator and information swapper [4]. Then, in terms to the construction of the Cloud of Mental Health Education in Colleges and Universities, educational innovation must absorb the essence of the traditional mental health education and meet the need of the physical and mental growth of college students.

\section{The elements of the Cloud of Mental Health EDUCATION IN COLLEGES AND UNIVERSITIES}

\section{A. The construction of the Cloud platforms}

That the platforms of the Cloud of Mental Health Education in Colleges and Universities is based on advanced technology equipment and excellent Cloud algorithm will attract enormous numbers of college students as its users. As is shown in figure 1, it's a consolidated bodies that has 4 parts which can interact with each other.

The Platform of the Cloud of Mental Health Education in Colleges and Universities

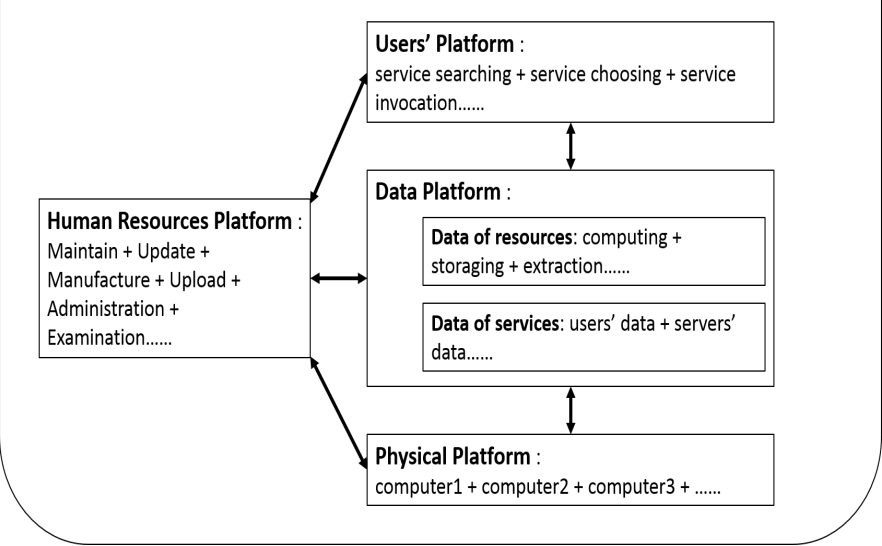

Fig. 1. Framework of the Platform of the Cloud of Mental Health Education in Colleges and Universities

1) Physical platform: The physical basics of the Cloud are computer clusters which have been constructed as an incredible data center. The Cloud of Mental Health Education in Colleges and Universities has connected the computers of administrative departments of education, psychology related departments in colleges and social organizations and institutions by Cloud computing technology which also can integrate the resources in those computers. Accordingly, the computer clusters are steady physical preparation for the extraction and access of resources [5].

2) Data platform: The data platform is the interlayer which is the bridge that combines physical platform with users' platform and its main function is to collect, store, exchange data. Generally, the date can be classified in to two categories: data of resources and data of services. The former can put he wide range of heterogeneous information resources together and remove redundant constructions and wipe the redundant data when it enriches resources constantly. The latter consist of receiving request data and providing service 
data. To go into greater detail, the proper management of data no matter in user end or in Cloud, acquisition of performance data and task scheduling can make two functions mentioned in great order and ensure the users have the access to anywhere of the Cloud.

3) User's platform: The construction of user's platform includes two tasks. Firstly, the potential user group of the Mental Health Education in Colleges and Universities can not be only meant for the college set, but the students' parents, mental health education workers and related social institutions. Secondly, the approaches to it will be designed in varied forms such as cross-platform compatible apps of phone or Pad and High-tech intelligent equipment with function of off-line data transference. And if so, there would be a win-win situation that users can enjoy the resources of the Cloud efficiently and conveniently, on the other hand, the Cloud can record and store users' behavioral date and preference to deliver wellmatched services.

4) Human resources platform: The construction of user's platform is of significant in whole plan. Although the powerful characteristics of Cloud computing technology can make the data and resources transfer safely and efficiently among those three platforms, notably, the participation of human resources is going to coordinate those three. Firstly, for physical platform, the maintenance of the equipment can not be realized without technicians. Secondly, for data platform, mental health education resources are made by professional mental health education workers and the upload rules used to audit and manage resources are carried out by the authorities. What's more, it is essential that scientific research teams are good at updating computer algorithm and patching security vulnerabilities. Thirdly, Restricting users' requests and taking intervention measures for high-risk users can not be absolutely automatic.

\section{B. The construction of the Cloud services}

It is vital that the Cloud of Mental Health Education in Colleges and Universities can provide various Cloud services that users can choose to resolve their own mental puzzles and learn the knowledge of the mental health education. Basically, the Cloud services can be divided into three categories.

1) Personalized service: Personalized service is aimed at the need of the individuals which facilitates users to select the resources they want. To the first, users can learn the common knowledge from the mental health education online video open class and the massive open online courses ${ }^{[6]}$. Secondly, the cloud can record and storage the mental growth of the college students which can be made into their mental health reports. In addition, A variety of scales (e.g., SCL-90, MMPI, MBTI, 16PF) are provided to users which can be used to evaluate their own mental health status.

2) Cooperative service: Cooperative service is similar to the $\mathrm{C} 2 \mathrm{C}$ model in E-business, which means that cooperation can be realized among users. For example, users can upload their own mental health education resources into the shared section of the Cloud which can be extracted by others conveniently. Moreover, Online chatting rooms and BBS would be used into compeer mental tutorship. Along with the popularity of the video 3D technology, affective communication across time and space may be around the corner [7].

3) Help service: Help service supplies another more professional way to solve the mental problems when college students can not resolve them by personalized and cooperative service. On the one hand, Users can make appointment of counseling or treatment with experts in the resource pool according to their own situation, on the other hand, experts can master the psychological development and change of the people who seeking for help and do real-time monitoring and timely intervention of the special individuals in case of the emergencies.

\section{CONCLUSION}

In present, the era is transferring to the big data with Cloud technology. The transmission and dissemination of data is becoming more and more mini, instantaneous and flat, while the storage and reception of information resources tend to be huge and systematic. Colleges and universities can not only stick to the traditional network psychological health education model, but should catch up with the advanced technologies. Therefore, building the Cloud of Mental Health Education in Colleges and Universities and transferring the traditional mental health education field into the technical supported Cloud platform can not only achieve the realize the sharing and efficient use of mental health resources, but also able to enhance the effect of mental health education through a wide range of Cloud services.

\section{ACKNOWLEDGEMENTS}

This paper was guided by Prof. Zeng Weixi (School of Political Science and Public Administration, University of Electronic Science and Technology of China) and Prof. Li Yuan (Mental Health Education Center, University of Electronic Science and Technology of China). I gratefully acknowledge their invaluable suggestions in preparing this paper. My sincere appreciation also goes to the teachers from the Mental Health Education Center of UESTC. In addition, I would like to thank the anonymous reviewers who have helped to improve the paper.

\section{REFERENCES}

[1] Yao Benxian, Lu Lu. Status and Prospect of the research on the education of mental health in China [J]. Psychological science, 2007, 30 (2), pp. 485-488.( In Chinese)

[2] Guo Dongmei, Meng Yan. Research on cloud computing and data storage and management technology [J]. Information technology, 2012 (5), pp. 73-76. ( In Chinese)

[3] Jiang Qiaoling, $\mathrm{Hu}$ Kai. Research progress and trend of College Students' network mental health education [J]. Modern university education, 2013 (6), pp. 81-86. ( In Chinese)

[4] Zhou Banghua. Analysis of the current situation of network ideological and political education in the new period [J]. People's Tribune. 2012 (35), pp. 154-155. ( In Chinese) 
[5] Zeng Wenxiong. Sharing one day: primary and secondary school mental health education in the age of the Cloud [J]. Mental health education in primary and secondary schools, 2012 (17), pp. 4-6. ( In Chinese)

[6] Liang Fangmei. Study on the teaching reform of mental health education for college students in the age of the Cloud era [J]. Asia Pacific Education, 2015 (8), pp. 86-86. ( In Chinese)
[7] Zeng Wenxiong. Teaching and management of mental health education in primary and secondary schools based on cloud computing [J]. Teaching and management: theoretical edition, 2013 (27), pp. 33-35. ( In Chinese) 